\title{
GLOBAL SOLVABILITY OF THE DERIVATIVE NONLINEAR SCHRÖDINGER EQUATION
}

\author{
JYH-HAO LEE
}

ABstract. The derivative nonlinear Schrödinger equation (DNLS)

$$
i q_{t}=q_{x x} \pm\left(q^{*} q^{2}\right)_{x}, \quad q=q(x, t), i=\sqrt{-1}, q^{*}(z)=\overline{q(z)},
$$

was first derived by plasma physicists $[9,10]$. This equation was used to interpret the propagation of circular polarized nonlinear Alfvén waves in plasma. Kaup and Newell obtained the soliton solutions of DNLS in 1978 [5]. The author obtained the local solvability of DNLS in his dissertation [6]. In this paper we obtain global existence (in time $t$ ) of Schwartz class solutions of DNLS if the $L^{2}$-norm of the generic initial data $q(x, 0)$ is bounded.

\section{INVERSE SCATTERING FOR A ZAKHAROV-SHABAT SYSTEM}

In order to prove the local solvability of the derivative nonlinear Schrödinger equation, the author [6] considered the following transformation:

$$
u_{t}=i u_{x x}+\varepsilon\left(u^{*} u^{2}\right)_{x}, \quad \varepsilon= \pm 1 \quad \text { (DNLS). }
$$

Let $q=u \exp \left(\int_{-\infty}^{x}-i \varepsilon u u^{*}\right)$. Then $q$ satisfies

$$
q_{t}=(i / 2) q_{x x}-(\varepsilon / 2) q^{2} q_{x}^{*}+(i / 4) q|q|^{4} .
$$

Equation (1.2) is solvable by the spectral problem $\frac{d m}{d x}=z^{2}[J, m]+z Q m+P m$, where $Q=\left(\begin{array}{cc}0 & q \\ \varepsilon q^{*} & 0\end{array}\right)$ and $P=Q(\operatorname{ad} J)^{-1} Q$; here ad $J(A) \equiv[J, A]$.

At first we consider a more general case. By a potential here we mean a pair of functions $(Q, P), Q, P: \mathbf{R} \rightarrow M_{2}(C)=$ set of $2 \times 2$ complex matrices, $Q$ is offdiagonal, and the diagonal part of $P$ equals $Q(\operatorname{ad} J)^{-1} Q, Q, Q_{x}, P, P_{x} \in L^{1}$. We consider the following spectral problem: given $z \notin \Sigma=\left\{z: \operatorname{Im}\left(z^{2}\right)=0\right\}$, find $m(\cdot, z): \mathbf{R} \rightarrow M_{2}(C)$ with

$$
\begin{gathered}
\frac{\partial}{\partial x} m(x, z)=z^{2}[J, m(x, z)]+z Q(x) m(x, z)+P(x) m(x, z), \\
m(\cdot, z) \text { bounded, } \quad m(x, z) \rightarrow I \quad \text { as } x \rightarrow-\infty, \\
\text { here } J=\left(\begin{array}{cc}
-i & 0 \\
0 & i
\end{array}\right), i=\sqrt{-1} .
\end{gathered}
$$

Received by the editors February 12, 1987.

1980 Mathematics Subject Classification (1985 Revision). Primary 35Q20, 35 Q 15.

Key words and phrases. Integrable evolution equation, inverse scattering transform, RiemannHilbert problem. 
Let $\Omega_{+}=\left\{z: \operatorname{Im}\left(z^{2}\right)>0\right\}$ and $\Omega_{-}=\left\{z: \operatorname{Im}\left(z^{2}\right)<0\right\}$. For a certain set of potentials, called generic, the solution $m$ has the following properties [6]:

For any $z \in \Sigma=\left\{z: \operatorname{Im}\left(z^{2}\right)=0\right\}$, there is a unique matrix $v(z)$ such that for all $x$

$$
m^{+}(x, z)=m^{-}(x, z) e^{x z^{2} J} v(z) e^{-x z^{2} J},
$$

where $m^{+}(x, z)=$ limit of $m$ on $\Sigma$ from $\Omega_{+}$and $m^{-}(x, z)=$ limit of $m$ on $\Sigma$ from $\Omega_{-}$;

$m(x, \cdot)$ has a finite number of poles at $z_{1}, z_{2}, \ldots, z_{N}$ (which do not depend on $x$ ), for any $z_{j}$ there is a matrix $v\left(z_{j}\right)$ such that

$$
\operatorname{Res}\left(m(x, \cdot), z_{j}\right)=\lim _{z \rightarrow z_{j}} m(x, z) e^{x z_{j}^{2} J} v\left(z_{j}\right) e^{-x z_{j}^{2} J}
$$

$$
\begin{aligned}
& \text { The map }(Q, P) \rightarrow V=\left\{v(z): z_{1}, z_{2}, \ldots, z_{N} ; v\left(z_{1}\right),\right. \\
& \left.v\left(z_{2}\right), \ldots, v\left(z_{N}\right)\right\} \text { is injective }
\end{aligned}
$$

We denote this map by sd i.e. $\operatorname{sd}(Q, P)=v$.

By a similar argument to [2], we can show the generic Schwartz class potentials form an open and dense set in Schwartz class potentials, i.e. those potentials for which every derivative is of rapid decrease. To make our exposition more clear, from now on we assume $(Q, P)$ is of Schwartz class. If $(Q, P)$ is generic, we call the associated function $v: \Sigma \cup D \rightarrow M_{n}(C)$ the scattering data, where $D=\left\{z_{1}, z_{2}, \ldots, z_{N}\right\}$. The scattering data satisfies the following constraints:

If $z_{j}$ is a pole, then $v\left(z_{j}\right)=c_{j}\left(\begin{array}{ll}0 & 1 \\ 0 & 0\end{array}\right)$ when $z_{j} \in \Omega_{+}$and $v\left(z_{j}\right)=c_{j}\left(\begin{array}{ll}0 & 0 \\ 1 & 0\end{array}\right)$ when $z_{j} \in \Omega_{-}$;

The winding number of $v_{22}(z)$ equals $\beta^{+}-\beta^{-}$, where $\beta^{+}$is

$$
\begin{gathered}
\operatorname{det}\left(\begin{array}{ll}
v_{11} & v_{12} \\
v_{21} & v_{22}
\end{array}\right)=1, \quad v_{11}=1 ; \\
v_{22}(z) \neq 0
\end{gathered}
$$
the number of $z_{j}$ in $\Omega_{+}$and $\beta^{-}$is the number of $z_{j}$ in $\Omega_{-}$, i.e. $\beta^{+}-\beta^{-}=\int_{\Sigma} d\left[\arg \left(v_{22}\right)\right]$ and $\Sigma$ is oriented such that $\Omega_{+}$ is in the left side;

Roughly speaking the smoothness of $(Q, P)$ implies the decay of $v(z)$ and the decay of $(Q, P)$ implies the smoothness of $v(z)$. These are aspects of Fourier-like theory, hence if $(Q, P)$ is a Schwartz class generic potential, then $v(z)-I$ is of Schwartz class. 
(1.13) Remark. Let $(Q, P)$ be a potential of compact support. Let $m_{0}(x, z)$ be the solution of the Volterra integral equation:

$$
m_{0}(x, z)=I+\int_{-\infty}^{x} e^{(x-y) z^{2} J}(z Q(y)+P(y)) m_{0}(y, z) e^{(y-x) z^{2} J} d y .
$$

The dependence on the parameter $z$ is holomorphic, so $m_{0}(x, \cdot)$ is an entire function, $m_{0}(\cdot, z)$ is absolutely continuous, $m_{0}(x, z)=I$ if $x \ll 0$ and $m_{0}(x, z)=e^{x z^{2} J} S(z) e^{-x z^{2} J}$ if $x \gg 0$. Let

$$
\begin{gathered}
S(z)=\left(\begin{array}{cc}
S_{11}(z) & S_{12}(z) \\
S_{21}(z) & S_{22}(z)
\end{array}\right) ; \\
a(z)=\left(\begin{array}{cc}
1 & -S_{12}(z) / S_{11}(z) \\
0 & 1
\end{array}\right) \quad \text { for } z \in \Omega_{+}, \\
a(z)=\left(\begin{array}{cc}
1 & 0 \\
-S_{12}(z) / S_{22}(z) & 1
\end{array}\right) \quad \text { for } z \in \Omega_{-} ;
\end{gathered}
$$

and

$$
m(x, z)=m_{0}(x, z) e^{x z^{2} J} a(z) e^{-x z^{2} J} .
$$

Then $m(x, z)$ satisfies (1.3) and (1.4). The poles of $m(x, \cdot)$ in $\Omega_{+}$is the zero set of $S_{11}(z)$ and the poles of $m(x, \cdot)$ in $\Omega_{-}$is the zero set of $S_{22}(z)$. Since $\lim _{|z| \rightarrow \infty} S_{11}(z)=\lim _{|z| \rightarrow \infty} S_{22}(z)=1$, the set $D=\left\{z: S_{11}(z)=0\right.$ or $\left.S_{22}(z)=0\right\}$ is finite. If $Z \cap \Sigma=\varnothing$ then $(Q, P)$ is generic. For a generic potential of compact support,

$$
\begin{gathered}
v(z)=\left(\begin{array}{cc}
1 & -S_{12}(z) / S_{11}(z) \\
S_{21}(z) / S_{22}(z) & 1-S_{21}(z) S_{12}(z) /\left(S_{11}(z) S_{22}(z)\right)
\end{array}\right) ; \\
v\left(z_{j}\right)=-S_{12}\left(z_{j}\right) / S_{11}^{\prime}\left(z_{j}\right)\left(\begin{array}{ll}
0 & 1 \\
0 & 0
\end{array}\right), \quad z_{j} \in \Omega_{+}, \\
v\left(z_{j}\right)=-S_{21}\left(z_{j}\right) / S_{22}^{\prime}\left(z_{j}\right)\left(\begin{array}{cc}
0 & 0 \\
1 & 0
\end{array}\right), \quad z_{j} \in \Omega_{-} .
\end{gathered}
$$

See [6].

Given a scattering data $v$ satisfying (1.8)-(1.12), the inverse problem amounts to solving an analytic factorization problem (Riemann-Hilbert problem) with one parameter $x$, i.e. we want to find $m(x, \cdot)$ that is meromorphic on $\mathbf{C} \backslash \Sigma$ such that

$$
\left\{\begin{array}{c}
m^{+}(x, z)=m^{-}(x, z) e^{x z^{2} J} v(z) e^{-x z^{2} J}, \quad z \in \Sigma, \\
\operatorname{Res}\left(m(x, z), z_{j}\right)=\lim _{z \rightarrow z_{j}} m(x, z) e^{x z_{j}^{2} J} v\left(z_{j}\right) e^{-x z_{j}^{2} J}, \\
j=1,2, \ldots, N ; \text { and } m(x, z) \rightarrow I \text { as }|z| \rightarrow \infty .
\end{array}\right.
$$

As shown in [6], the inverse problem is solvable if $v(z)-I$ is small (i.e. $\|v-I\|_{\infty}<\varepsilon$ for some small number $\varepsilon$ ). If $v(z)-I$ is not small, we choose 
a piecewise rational function $u$ with the following properties:

(i) $\quad u_{j j}=1, u(z)$ is upper triangular in $\Omega_{+}$and lower triangular in $\Omega_{-}$respectively;

(ii) $u(z) \rightarrow I \quad$ as $|z| \rightarrow \infty$;

(iii) $\left\|u^{-} v\left(u^{+}\right)^{-1}-I\right\|_{\infty}<\varepsilon \quad[2,6]$.

We let $v^{\sharp}=u^{-} v\left(u^{+}\right)^{-1}$ since $v^{\sharp}-I$ is small, and $v^{\sharp}$ also satisfies (1.8) and (1.12). Hence we obtain an associated eigenfunction $m^{\sharp}$; we may look for $m$ in the form $m(x, z)=r(x, z) m^{\sharp}(x, z) e^{x z^{2} J} u(z) e^{-x z^{2} J}$. Let

$$
r(x, z)=I+\sum_{k=1}^{p}\left(z-z_{k}\right)^{-1} a_{k}(x) .
$$

The conditions (1.20) amount to a system of linear equations of the residues $a_{k}(x)$ of $r$.

Let $\mathrm{SD}=$ set of functions $v=\left(v, z_{1}, z_{2}, \ldots, z_{N} ; v\left(z_{1}\right), \ldots, v\left(z_{N}\right)\right)$ which satisfy the conditions (1.8)-(1.12). The set of $v \in \mathrm{SD}$ such that $m$ is solvable is open and dense in $\mathrm{SD}$; such $v$ we call generic. Note that $v \in \mathrm{SD}$ is generic if and only if there exists a potential $(Q, P)$ such that $\operatorname{sd}(Q, P)=v$. Let $m \sim I+m_{1} / z+m_{2} / z^{2}+\cdots$,

$$
Q=-\left[J, m_{1}\right], \quad P=-Q m_{1}-\left[J, m_{2}\right] .
$$

Then $\frac{d m}{d x}=z^{2}[J, m]+z Q m+P m, m(\cdot, z)$ bounded, and $m(x, z) \rightarrow I$ as $x \rightarrow-\infty$. Symbolically we have

$$
v \rightarrow m \rightarrow(Q, P),
$$

(scattering data) $\rightarrow$ (eigenfunction) $\rightarrow$ (potential).

If $Q=\left(\begin{array}{cc}0 & q \\ \varepsilon q^{*} & 0\end{array}\right)$, and the off-diagonal part of $P=0$, then the scattering data $v$ has extra conditions:

$$
(v(\varepsilon \bar{z}))^{*}=v(z), \quad\left(v\left(\varepsilon \bar{z}_{j}\right)\right)^{*}=-v\left(z_{j}\right), \quad \varepsilon= \pm 1 .
$$

Let $\sigma$ be an automorphism on $M_{2}(C)$ defined by $\left(\begin{array}{ll}a & b \\ c & d\end{array}\right)^{\sigma}=\left(\begin{array}{cc}a & -b \\ -c & d\end{array}\right)$. Since $Q^{\sigma}=-Q$ and $P^{\sigma}=P$ we have

$$
v^{\sigma}(-z)=v(z), \quad v^{\sigma}\left(-z_{j}\right)=-v\left(z_{j}\right) .
$$

Conversely if $v(z) \in \mathrm{SD}$ and satisfies (1.23) and (1.24), and $v$ is generic, then the associated potential $(Q, P)$ satisfies the following properties: $Q=\left(\begin{array}{c}0 \\ \varepsilon q^{*} \\ q\end{array}\right)$, i.e. $Q^{*}=\varepsilon Q$, the off-diagonal part of $P=0$, and the diagonal part of $P=$ $Q(\operatorname{ad} J)^{-1} Q$.

If $v(z, t)$ satisfies the evolution equation

$$
\left\{\begin{aligned}
& d v(z, t) / d t= z^{k-1}(J, v(z, t)), \\
& k \text { is an odd positive integer } ; \\
& d v\left(z_{j}, t\right) / d t= z_{j}^{k-1}\left(J, v\left(z_{j}, t\right)\right), \\
& z_{j} \text { is fixed for each } j,
\end{aligned}\right.
$$


and if $v(z, 0)$ is generic in $\mathrm{SD}$, then $v(z, t)$ is generic for $t$ small (since the set of the generic potentials is open in SD). The corresponding potential $(Q(x, t), P(x, t))$ satisfies the evolution equation

$$
Q_{t}=\left[J, \lambda_{k}\right], \quad P_{t}=\left[Q, \lambda_{k}\right]+\left[J, \lambda_{k+1}\right],
$$

where $\lambda_{k}=\lambda_{k}(Q, P)$ are computed by the recurrence formula $d \lambda_{k} / d x-$ $\left[P, \lambda_{k}\right]=\left[Q, \lambda_{k+1}\right]+\left[J, \lambda_{k+2}\right], \lambda_{0}=J$.

If $v(z, 0)$ satisfies (1.23) and (1.24) and $k=5$, then equation (1.26) is reduced to

$$
q_{t}=(i / 2) q_{x x}-(\varepsilon / 2) q^{2} q_{x}^{*}+q|q|^{4}
$$

Note that $Q=\left(\begin{array}{cc}0 & q \\ \varepsilon q^{*} & 0\end{array}\right)$ and $\left(Q, Q(\operatorname{ad} J)^{-1} Q\right)$ is the associated potential with respect to the scattering data $v(z, t)$. Let $u=q \exp \left(\int_{-\infty}^{x} i \varepsilon q q^{*}\right)$. Then $u$ satisfies

$$
u_{t}=i u_{x x}+\varepsilon\left(u^{*} u^{2}\right)_{x}
$$

This is the derivative nonlinear Schrödinger equation (DNLS).

Let $E=\left\{z_{1}, z_{2}, \ldots, z_{N}\right\}, z_{i} \notin \Sigma$ and $z_{1}, z_{2}, \ldots, z_{N}$ are distinct points. Denote $\operatorname{SD}(E)=\{v \in \mathrm{SD}: v$ is a function on $\Sigma \cup E\}$.

Theorem A. Suppose $\left(Q_{0}, P_{0}\right)$ is a generic Schwartz class potential and suppose $t \rightarrow v(\cdot, t)$ is a smooth map from $[0, \infty)$ to $\operatorname{SD}(E)$ such that $v(\cdot, 0)=$ $\operatorname{sd}\left(Q_{0}, P_{0}\right)$. Then there is $a T>0$ and a unique smooth map

$$
t \rightarrow(Q(\cdot, t), P(\cdot, t))
$$

from $[0, T)$ to Schwartz class potentials such that $\operatorname{sd}(Q(\cdot, t), P(\cdot, t))=v(\cdot, t)$, $t \in[0, T)$.

Proof. Since the set of generic formal scattering data is open, $v(\cdot, t)$ remains generic over some nonempty interval $[0, T)$. The corresponding potentials $(Q(\cdot, t), P(\cdot, t))=\operatorname{sd}^{-1}(v(\cdot, t))$ are uniquely determined. $(Q(\cdot, t), P(\cdot, t))$ is smooth by our construction, see $[2,3,6]$.

Theorem B. Suppose $q(x, t)$ satisfies DNLS, i.e. $q_{t}=i q_{x x}+\varepsilon\left(|q|^{2} q\right)_{x}, \varepsilon= \pm 1$, and $t \rightarrow q(\cdot, t)$ is smooth from $[0, T)$ to a Schwartz class. Then

$$
\int_{-\infty}^{\infty}|q(x, t)|^{2} d x=\int_{-\infty}^{\infty}|q(x, 0)|^{2} d x,
$$

i.e. the $L^{2}$ norm of $q(\cdot, t)$ is invariant under DNLS.

Proof. $|q|_{t}^{2}=\left(q q^{*}\right)_{t}=q_{t} q^{*}+q q_{t}^{*}=i\left(q_{x} q^{*}-q_{x}^{*} q\right)_{x}+3 \varepsilon / 2|q|_{x}^{4}$. Hence

$$
\frac{d}{d x} \int_{-\infty}^{\infty}|q(x, t)|^{2} d x=\left.\left(i\left(q_{x} q^{*}-q_{x}^{*} q\right)+\frac{3 \varepsilon}{2}|q|^{4}\right)\right|_{-\infty} ^{\infty}=0
$$

Theorem $\mathbf{B}^{\prime}$. Suppose $q(x, t)$ satisfies (1.2), i.e. $q_{t}=(i / 2) q_{x x}-(\varepsilon / 2) q^{2} q_{x}^{*}+$ $\left.(1 / 4) q|q|\right|^{4}$, and $t \rightarrow q(\cdot, t)$ is smooth from $[0, T)$ to a Schwartz class. Then

$$
\int_{-\infty}^{\infty}|q(x, t)|^{2} d x=\int_{-\infty}^{\infty}|q(x, 0)|^{2} d x
$$


Proof. Let $u=q \exp \left(\int_{-\infty}^{x} i \varepsilon q q^{*}\right)$. Then $u$ satisfies DNLS, hence

$$
\int_{-\infty}^{\infty}|u(x, t)|^{2} d x=\int_{-\infty}^{\infty}|u(x, 0)|^{2} d x
$$

Note that $u u^{*}=q q^{*}$, hence $\int_{-\infty}^{\infty}|q(x, t)|^{2} d x=\int_{-\infty}^{\infty}|q(x, 0)|^{2} d x$.

Theorem C. Let $Q_{0}=\left(\begin{array}{cc}0 & q_{0} \\ \varepsilon q_{0} & 0\end{array}\right)$. If $\left(Q_{0}, Q_{0}(\operatorname{ad} J)^{-1} Q_{0}\right)$ is a generic Schwartz class potential, then there exists unique $q(x, t), 0 \leq t<\infty$, such that

$$
\left\{\begin{array}{l}
q_{t}=(i / 2) q_{x x}-(\varepsilon / 2) q^{2} q_{x}^{*}+(i / 4) q|q|^{4}, \\
q(x, 0)=q_{0} .
\end{array}\right.
$$

Theorem $\mathbf{C}^{\prime}$. If $u_{0}(x)$ is of Schwartz class, $q_{0}=u_{0} \exp \left(\int_{-\infty}^{x}-i \varepsilon u_{0} u_{0}^{*}\right), Q_{0}=$ $\left(\begin{array}{cc}0 & q_{0} \\ \varepsilon q_{0} & 0\end{array}\right)$, and $\left(Q_{0}, Q_{0}(\operatorname{ad} J)^{-1} Q_{0}\right)$ is generic (i.e. there exists $v$ such that

$$
\left.\operatorname{sd}\left(Q_{0}, Q_{0}(\operatorname{ad} J)^{-1} Q_{0}\right)=v\right),
$$

then there exists unique $u(x, t), 0 \leq t<\infty$, such that

$$
u_{t}=i u_{x x}+\varepsilon\left(|u|^{2} u\right)_{x}, \quad u(x, 0)=u_{0}(x) .
$$

Proof. This follows from Theorem C.

\section{Derivation of the eVolution equation}

Now we derive the evolution equation (1.26). We use the $\bar{\partial}$ idea of R. Beals and R. R. Coifman [2,3]. For fixed $x$ the eigenfunction $m(x, \cdot)$ for a generic potential can be considered as a matrix-valued tempered distribution on $\mathbb{C}$. $\partial m / \partial \bar{z}$ is a tempered distribution supported on $\Sigma \cup\left\{z_{1}, z_{2}, \ldots, z_{N}\right\}$.

(2.1) Lemma. If $f$ is in $L^{\infty}(\mathbb{C})$ and $\partial f / \partial \bar{z}=\mu$ vanishes rapidly at $\infty$, then $f$ has an asymptotic expansion

$$
f(z)-f_{0}+f_{1} / z+f_{2} / z^{2}+\cdots, \quad f_{k}=\int_{k>1}-z^{k-1} d \mu(z),
$$

in the sense that

$$
\left|f(z)-\sum_{j=0}^{N-1} f_{j} z^{-j}\right| \leq C_{N}|z|^{-N} \operatorname{dist}(z, \operatorname{supp} \mu)^{-1}
$$

In particular if $v(z)-I$ decays rapidly on $\Sigma$, the associated eigenfunction $m$ has an asymptotic expansion in $z$,

$$
m(x, z) \sim m_{0}+m_{1} / z+m_{2} / z^{2}+\cdots, \quad z \rightarrow \infty, m_{0}=I .
$$

Since $d m / d x=z^{2}[J, m]+z Q m+p m$, we see that

$$
(d / d x-p) m_{k}=Q m_{k+1}+\left[J, m_{k+2}\right], \quad k=0,1,2, \ldots
$$

Especially

$$
Q=-\left[J, m_{1}\right], \quad P=-Q m_{1}-\left[J, m_{2}\right]
$$


Now suppose that the evolution of the scattering data is given by

$$
\begin{aligned}
d v(z, t) / d t & =z^{k-1}[J, v(z, t)], \\
d v\left(z_{j}, t\right) / d t & =z_{j}^{k-1}\left[J, v\left(z_{j}, t\right)\right],
\end{aligned}
$$

and $z_{j}$ is fixed for each $j$. Then $m$ and $Q, P$ also evolve in $t$. We denote $t$-differentiation by a dot. Differentiating (2.4) with respect to the $t$-variable we obtain

$$
\dot{Q}=-\left[J, \dot{m}_{1}\right], \quad \dot{P}=-\dot{Q} m_{1}-Q \dot{m}_{1}-\left[J, \dot{m}_{2}\right] .
$$

Since $m$ has an asymptotic expansion, we have $m J m^{-1} \sim J+\lambda_{1} / z+\lambda_{2} / z^{2}+$ $\cdots$, where $\lambda_{k}=\lambda_{k, Q, P}$ and $\dot{m} m^{-1} \sim f_{1} / z+f_{2} / z^{2}+\cdots$, where $f_{k}(x, t)=$ $\int z^{k-1}\left[\partial\left(\dot{m} m^{-1}\right) / \partial \bar{z}\right]$. Then $\dot{m}=\left(\dot{m} m^{-1}\right) m \sim f_{1} / z+\left(f_{1} m_{1}+f_{2}\right) / z^{2}+\cdots$. Hence

$$
\dot{m}_{1}=f_{1}, \quad \dot{m}_{2}=f_{1} m_{1}+f_{2} .
$$

Substituting (2.7) into (2.6) we get

$$
\dot{Q}=-\left[J, f_{1}\right], \quad \dot{P}=-\left[Q, f_{1}\right]-\left[J, f_{2}\right] .
$$

We see that $\partial\left(\dot{m} m^{-1}\right) / \partial \bar{z}$ and $z^{k-1} \partial\left(m J m^{-1}\right) / \partial \bar{z}$ are both supported on $\Sigma \cup\left\{z_{1}, z_{2}, \cdots, z_{N}\right\}$. By the argument in [3],

$$
\partial\left(\dot{m} m^{-1}\right) / \partial \bar{z}=z^{k-1} \partial\left(m J m^{-1}\right) / \partial \bar{z} .
$$

Hence $f_{1}=-\lambda_{k}$ and $f_{2}=-\lambda_{k+1} \cdot(Q, P)$ evolves as

$$
\dot{Q}=\left[J, \lambda_{k}\right], \quad \dot{P}=\left[Q, \lambda_{k}\right]+\left[J, \lambda_{k+1}\right] .
$$

\section{ESTIMATE OF $m$ IN $L^{2}$-NORM OF THE POTENTIAL}

As we show below, the technique used in $[2,6]$ to solve the inverse problem reduces solvability at $t=T$ to control $m(x, t, z)$ as $t \rightarrow T$. Recall that the equation for $m$ is

$$
\frac{d m}{d x}=z^{2}[J, m]+z Q m+P m,
$$

where $Q=\left(\begin{array}{cc}0 & q \\ \varepsilon q^{*} & 0\end{array}\right)$ and $P=Q(\operatorname{ad} J)^{-1} Q$. Let $\tilde{m}=\exp \left(\int_{-\infty}^{x} P\right) m$. Then $\tilde{m}$ satisfies

$$
\frac{d \tilde{m}}{d x}=z^{2}[J, \tilde{m}]+Q \tilde{m},
$$

where

$$
\widetilde{Q}=\exp \left(-\int_{-\infty}^{x} Q(\operatorname{ad} J)^{-1} Q\right) Q \exp \left(\int_{-\infty}^{x} Q(\operatorname{ad} J)^{-1} Q\right)
$$


Since $Q(\operatorname{ad} J)^{-1} Q$ is purely imaginary,

$$
\begin{gathered}
\left|m_{i j}(x, z)\right|=\left|\tilde{m}_{i j}(x, z)\right| ; \quad\|Q\|_{2}=\|\widetilde{Q}\|_{2} ; \\
\left|\lim _{x \rightarrow \infty} m_{i j}(x, z)\right|=\left|\lim _{x \rightarrow \infty} \tilde{m}_{i j}(x, z)\right| .
\end{gathered}
$$

Writing $m$ instead of $\tilde{m}$, we may consider the following problem:

$$
\frac{d}{d x} m=z^{2}[J, m]+\left(\begin{array}{ll}
0 & q \\
r & 0
\end{array}\right) m,
$$

here $J=\left(\begin{array}{cc}-i & 0 \\ 0 & i\end{array}\right), i=\sqrt{-1}$. Taking the first column of $m$, we have

$$
\frac{d}{d x}\left(\begin{array}{l}
m_{11} \\
m_{21}
\end{array}\right)=z^{2}\left(\begin{array}{c}
0 \\
(2 i) m_{12}
\end{array}\right)+\left(\begin{array}{ll}
0 & q \\
r & 0
\end{array}\right)\left(\begin{array}{l}
m_{11} \\
m_{21}
\end{array}\right),
$$

i.e.

$$
\begin{aligned}
& \frac{d m_{11}}{d x}=q m_{12}, \quad m_{11}(-\infty)=1, m_{12}(-\infty)=0, \\
& \frac{d m_{12}}{d x}=z^{2}(2 i) m_{12}+r m_{11} .
\end{aligned}
$$

We are led to consider the following basic system of O.D.E.:

$$
\frac{d u}{d x}=q v, \quad \frac{d v}{d x}+c v=r u, \quad u(-\infty)=\alpha, v(-\infty)=0, c>0
$$

(3.7) Basic Lemma. Given $q, r \in L^{2}(-\infty, \infty)$, there are unique absolutely continuous bounded functions $u, v$ satisfying (3.6) and $\|u\|_{\infty}+\|v\|_{\infty} \leq F=$ $F\left(\|q\|_{2},\|r\|_{2},|\alpha|\right)$.

Proof. First we convert the equation (3.6) into an integral equation:

$$
u(x)=\alpha+\int_{-\infty}^{x} q v, \quad v(x)=\int_{-\infty}^{x} e^{c(y-x)} r u, \quad c>0 .
$$

At first we look for the solution $u, v$, where $u \in L^{2}$ and $v \in L^{\infty}$. Consider the following recurrence formula:

$$
u_{n}=\alpha+\int_{-\infty}^{x} q v_{n}, \quad v_{n+1}=\int_{-\infty}^{x} e^{c(y-x)} r u_{n}, \quad v_{0} \in L^{2} \text { arbitrary. }
$$

We have

$$
\begin{gathered}
\left\|u_{n+1}-u_{n}\right\|_{\infty} \leq\left(\|q\|_{2}\|r\|_{2} / c\right)\left\|u_{n}-u_{n-1}\right\|_{\infty} \\
\left\|u_{n+1}-u_{n}\right\| \leq\left(\|q\|_{2}\|r\|_{2} / c\right)\left\|u_{n}-u_{n-1}\right\|_{2} .
\end{gathered}
$$

Consider the following two cases:

Case 1. $\|q\|_{2}\|r\|_{2} / c<1$. Then $\left\{u_{n}\right\}$ is a Cauchy sequence in $L^{2}$ and $\left\{v_{n}\right\}$ is a Cauchy sequence in $L^{2}$; there exist $u \in L^{\infty}$ and $v \in L^{2}$ such that $u_{n} \rightarrow u$ in $L^{\infty}, v_{n} \rightarrow v$ in $L^{2}$ and

$$
\begin{gathered}
\left\|u-u_{0}\right\|_{\infty} \leq\left(1 /\left(1-\|q\|_{2}\|r\|_{2} / c\right)\right)\left\|u-u_{0}\right\|_{\infty}, \\
\left\|v-v_{0}\right\|_{2} \leq\left(1 /\left(1-\|q\|_{2}\|r\|_{2} / c\right)\right)\left\|v_{1}-u_{0}\right\|_{2} .
\end{gathered}
$$


Taking the limits of $u_{n}$ and $v_{n}$ in (3.9) we have

$$
u=\alpha+\int_{-\infty}^{x} q v d y, \quad v=\int_{-\infty}^{x} e^{c(y-x)} r u d y .
$$

Obviously $u, v$ are absolutely continuous and $u, v$ satisfy the O.D.E. (3.6). Note that

$$
h(x)= \begin{cases}e^{c x}, & x<0 \\ 0, & x \geq 0\end{cases}
$$

is also in $L^{2}$, hence $\|v\|_{\infty} \leq(1 / \sqrt{2 c})\|r\|_{2}\|u\|_{\infty}$.

Case 2. $\|q\|_{2}\|r\|_{\infty} / c<\infty$. Let $N$ be a positive integer satisfying

$$
\|q\|_{2}\|r\|_{2} /(N c)<1
$$

There exist a finite number of points $0=x_{0}, x_{1}, x_{2}, \ldots, x_{N}=\infty$ such that $\left(\int_{x_{j}}^{x_{j+1}}|q|^{2}\right)^{1 / 2} \leq\|q\|_{2} / N$. By Case 1 , the solutions $u, v$ exist up to the point $x_{1}$. Then consider the following equation with initial values at $x_{1}$ :

$$
\begin{aligned}
& u=u\left(x_{1}\right)+\int_{x_{1}}^{x} q v d y, \\
& v=e^{c\left(-x+x_{1}\right)} v\left(x_{1}\right)+\int_{x_{1}}^{x} e^{c(y-x)} r u d y,
\end{aligned}
$$

where $u\left(x_{1}\right)$ and $v\left(x_{1}\right)$ are constants which depend only on $\alpha,\|q\|_{2}$ and $\|r\|_{2}$. Since

$$
\left(\int_{x_{1}}^{x_{2}}|q|^{2}\right)^{1 / 2}\|r\|_{2} / c \leq\|q\|_{2}\|r\|_{2} /(N c)<1,
$$

again by Case 1 , we may extend the solution $u, v$ to the point $x_{2}$. If we continue, we obtain $u, v$, defined on the whole line and satisfying equation (3.6), $\|u\|_{\infty}+\|v\|_{\infty} \leq F=F\left(\|q\|_{2},\|r\|_{2},|\alpha|\right)<\infty$. We are done.

(3.12) Theorem. Assume $Q=\left(\begin{array}{ll}0 & q \\ r & 0\end{array}\right)$ is of Schwartz class. If $z$ is large enough and $1 / 2 \leq|\operatorname{Re}(z)| /|\operatorname{Im}(z)| \leq 2$, then there is a unique $m(x, z)$ which satisfies (3.3), (3.4), i.e.

$$
\begin{aligned}
& \frac{d}{d x} m=z^{2}[J, m]+Q m, \\
& m(\cdot, z) \text { bounded, } \quad m(x, z) \rightarrow I \quad \text { as } x \rightarrow-\infty
\end{aligned}
$$

Let

We have

$$
m=\left(\begin{array}{ll}
m_{11} & m_{12} \\
m_{21} & m_{22}
\end{array}\right)
$$

$$
\left\|m_{i j}(\cdot, z)\right\|_{\infty} \leq F=F\left(\|q\|_{2},\|r\|_{2},|d(z)|,|a(z)|\right),
$$

where $\lim _{x \rightarrow+\infty} m_{22}(x, z)=d(z)$ and $\lim _{x \rightarrow+\infty} m_{11}(x, z)=a(z)$.

Proof. The existence of $m(x, z)$ was proved in [6]. It suffices to estimate $m$ by the $L^{2}$-norm of $q, r$. We may assume $\operatorname{Im}\left(z^{2}\right)>0$. The first column of $m$ 
satisfies

$$
\begin{aligned}
& m_{11}(x, z)=\int_{-\infty}^{x} z q(y) m_{21}(y, z) d y+1, \\
& m_{21}(x, z)=\int_{-\infty}^{x} e^{(y-x)\left(-2 i z^{2}\right)} z r(y) m_{11}(y, z) d y .
\end{aligned}
$$

Multiplying by $z$ on both sides of the second equation of (3.13), we have

$$
\begin{aligned}
& m_{11}(x, z)=\int_{-\infty}^{x} q\left(z m_{21}(y, z)\right) d y+1, \\
& z m_{21}(x, z)=\int_{-\infty}^{x} e^{(y-x)\left(-2 i z^{2}\right)} z^{2} r(y) m_{11}(y, z) d y .
\end{aligned}
$$

Let $m_{11}=u$ and $z m_{21}=v$. If $1 / 2 \leq|\operatorname{Re}(z)| /|\operatorname{Im}(z)| \leq 2$, we have the same estimate for $m_{11}$ and $z m_{21}$; then

$$
\left\|m_{11}(\cdot, z)\right\|_{\infty}+\left\|z m_{21}(\cdot, z)\right\|_{\infty} \leq F\left(\|q\|_{2},\|r\|_{2}\right) .
$$

Hence $\left\|m_{11}(\cdot, z)\right\|_{\infty}+\left\|m_{21}(\cdot, z)\right\|_{\infty} \leq F\left(\|q\|_{2},\|r\|_{2}\right)$ if $|z|>1$. For the second column of $m$ we write the integral equation normalized at $\infty$. Note that $\lim _{x \rightarrow \infty} m_{22}(x, z)=d(z)$ and $\lim _{x \rightarrow \infty} m_{12}(x, z)=0$ (see [6, p. 33]),

$$
\begin{aligned}
& m_{22}(x, z)=d(z)-\int_{x}^{\infty} q(y)\left(z m_{12}(y, z)\right) d y, \\
& z m_{12}(x, z)=-\int_{x}^{\infty} e^{(y-x)\left(-2 i z^{2}\right)} z^{2} r(y) m_{22}(y, z) d y .
\end{aligned}
$$

Then by a similar argument we have the estimate

$$
\left\|m_{12}(\cdot, z)\right\|_{\infty}+\left\|m_{22}(\cdot, z)\right\|_{\infty} \leq F\left(\|q\|_{2},\|r\|_{2},|d(z)|\right) .
$$

Note that $\lim _{|z| \rightarrow \infty} d(z)=1$ and $\lim _{|z| \rightarrow \infty} a(z)=1$.

(3.16) Remarks. The point of Theorem (3.12) is not the existence of $m$, which was proved in [6]. As we show in [6],

$$
\begin{gathered}
\lim _{x \rightarrow \infty} m(x, z)=\delta(z)=\left(\begin{array}{cc}
a(z) & 0 \\
0 & d(z)
\end{array}\right) ; \\
\lim _{|z| \rightarrow \infty} a(z)=1, \quad \lim _{|z| \rightarrow \infty} d(z)=1,
\end{gathered}
$$

$m(\cdot, z)$ is bounded for $|z|>N$, where $N$ depends only on $d(z)$. The crucial fact here is that in the case $r=\varepsilon q^{*},\|q\|_{2}$ and $d(z)$ are invariant under the DNLS evolution.

Proof of Theorem C. By local solvability, the solution $q(t, x)$ exists for $0 \leq$ $t<T, T>0$. Let $Q=\left(\begin{array}{cc}0 & q \\ \varepsilon q^{*} & 0\end{array}\right), \operatorname{sd}\left(Q, Q(\operatorname{ad} J)^{-1} Q\right)=v(\cdot, t)$. According to Theorem (3.12), there is an open set $\Omega=\{z: 1 / 2<|\operatorname{Im}(z)| / \operatorname{Re}(z)|<2| z \mid,>$ $\left.C_{1}\right\}$ such that

$$
\left\|m\left(x, z, t_{\nu}\right)\right\| \leq C_{2} \quad \text { for all } x \in R, z \in \Omega,
$$

and for $\left\{t_{\nu}\right\}$ some sequence converging to $T$. Since $\{v(\cdot, t): 0 \leq t \leq T\}$ is bounded, we can choose the piecewise rational function $u(\cdot, t)$ of $(1.21)$ to 
depend smoothly on $t$ and to have simple poles in a fixed finite set $E^{\prime}$ independent of $t$. Recall $v^{\sharp}=u^{-} v\left(u^{+}\right)^{-1}$ and $m^{\sharp}$ is the eigenfunction associated to $v^{\sharp}$ :

$$
\left\|m^{\sharp}(x, t, \cdot)\right\| \leq C_{3} \quad \text { for }|z| \geq C_{4} .
$$

We look for $m(x, t, \cdot)$ of the form

$$
m(x, t, \cdot)=r(x, t, \cdot) m^{\sharp}(x, t, \cdot) e^{x z^{2} J} u(x, t, \cdot) e^{-x z^{2} J},
$$

where $r(x, t, \cdot)$ is rational with simple poles on $E \cup E^{\prime}$ and approaches $I$ at $z=\infty$.

$$
r(x, t, z)=I+\sum_{k=1}^{p}\left(z-z_{k}\right)^{-1} a_{k}(x, t),
$$

where $a_{k}(x, t)$ satisfies some system of linear equations and depends smoothly on $x$ and $t$. Moreover, for each $x$ and $t$ these equations have at most one solution, while the existence of a solution for all $x$ is the necessary and sufficient condition that $v(\cdot, t)$ be generic. Now $\operatorname{det} m^{\sharp}=1$ and $\operatorname{det}\left(e^{x z^{2} J} u(z) e^{-x z^{2} J}\right)=$ 1 , so (3.17), (3.18), (3.19) give

$$
\left\|r\left(x, t_{\nu}, z\right)\right\| \leq C_{5} \quad \text { for } x \in R, z \in \Omega,|z| \geq C_{4} .
$$

Passing to a subsequence, we deduce that $r\left(x, t_{\nu}, \cdot\right) \rightarrow r(x, T, \cdot)$, where the residues of $r(x, T, \cdot)$ solve the requisite linear equation at $t=T$. Therefore $v(\cdot, T)$ is generic. Since the set of generic data is open in SD, the solution $q(x, t)$ of the equation (1.20) exists for $0 \leq t<T+\varepsilon$. Obviously this implies the global solvability of the equation (1.27).

Acknowledgement. The author would like to thank Professor R. Beals and R. R. Coifman who suggested how to estimate $u, v$ in the Basic Lemma when $\|q\|_{2}$, $\|r\|_{2}$ are not small. The critical results of this paper were obtained when the author stayed at Yale University in April 1986. The author would like to thank Professor R. Beals for his hospitality.

\section{REFERENCES}

1. R. Beals and R. R. Coifman, Scattering transformation spectrales, et equations d'evolution non linearies, Seminaire Goulaouic-Meyer-Schwartz 1980-1981, exp. 22, Ecole Polytechnique, Palaiseau, 1981.

2. __ Scattering and inverse scattering for first order system, Comm. Pure Appl. Math. 37 (1984), 39-90.

3. __ Inverse scattering and evolution equations, Comm. Pure Appl. Math. 38 (1985), 29-42.

4. R. K. Bullough and P. J. Caudrey (eds.), Solitons, Topics in Current Physics, No. 17, SpringerVerlag, 1980.

5. D. J. Kaup and A. C. Newell, An exact solution for a derivative nonlinear Schrödinger equation, J. Math. Phys. 19 (1978), 789-801.

6. J. H. Lee, Analytic properties of Zakharov-Shabat inverse scattering problem with polynomial spectral dependence of degree 1 in the potential, Ph. D. Dissertation, Yale University, 1983. 
7. _ A Zakharov-Shabat inverse scattering problem and the associated evolution equations, Chinese J. Math. 12 (1984), 223-233.

8. __ Hamiltonian structure of soliton equations, Proc. CCNAA-AIT Seminar on Differential Equations (Taiwan, June 1985), pp. 205-214.

9. W. Mio et al., Modified nonlinear Schrödinger equation for Alfven waves propagating along the magnetic field in cold plasmas, J. Phys. Soc. Japan 41 (1976), 265-271.

10. E. Mjølhus, On the modulational instability of hydromagnetic waves parallel to the magnetic field, J. Plasma Phys. 16 (1976), 321-334.

11. V. E. Zakharov and A. B. Shabat, A refined theory of two-dimensional self-focussing and onedimensional self-modulation of waves in non-linear media, Soviet Phys. JETP 34 (1972), 62-69.

Institute of Mathematics, Academia Sinica, Taipei, Taiwan, Republic of China 